\title{
Two Generalized Predator-Prey Models for Integrated Pest Management with Stage Structure and Disease in the Prey Population
}

\author{
Ruiqing Shi, ${ }^{1,2}$ Sanyi Tang, ${ }^{1}$ and Wenli Feng ${ }^{2}$ \\ ${ }^{1}$ College of Mathematics and Information Science, Shaanxi Normal University, Xian, Shaanxi 710062, China \\ ${ }^{2}$ School of Mathematics and Computer Science, Shanxi Normal University, Linfen, Shanxi 041004, China \\ Correspondence should be addressed to Ruiqing Shi; shirq1979@163.com
}

Received 22 November 2012; Accepted 17 December 2012

Academic Editor: Jinhu Lü

Copyright (C) 2013 Ruiqing Shi et al. This is an open access article distributed under the Creative Commons Attribution License, which permits unrestricted use, distribution, and reproduction in any medium, provided the original work is properly cited.

Stage-structured predator-prey models with disease in the prey are constructed. For the purpose of integrated pest management, two types of impulsive control strategies (impulsive release of infective prey and impulsive release of predator) are used. For Case 1, infective prey applications are more frequent than releases of predator (natural enemies). For Case 2, predator (natural enemies) releases are more frequent than infective prey applications. In both cases, we get the sufficient conditions for the global attractivity of the susceptible prey-eradication periodic solution. In addition, the persistence of the systems is also discussed. At last, the results are discussed and some possible future work is put forward.

\section{Introduction}

Pests, such as insects, mice and other animals, unwanted plants (weeds), fungi, microorganisms, and so forth, are living organisms that occur where they are not wanted or that cause damage to crops or other animals. How to minimize the loss caused by the injurious insects and injurious germ carrier to the important plants, animals, and human being is always the common problem concerned by the entomologists and society. Human has adopted some advanced and modern weapons such as chemical pesticides, biological pesticides, remote sensing and measuring, and so on to deal with pests, and some great achievements have been obtained [1-7].

The traditional chemical control only care about the current effect, but seldom take the influence on the ecosystem into consideration. And it caused many problems such as environment pollution, pest resistance to the pesticide and pest reemergence, and the like. In this regard, it has been observed that beneficial insects are often more susceptible to chemical pesticides than the target pests are. In the same time, the concentration of the pesticides in use tends to increase with time and usage, since many pests develop resistance to these chemicals. This kind of pest management strategy was considered by many authors [8-12]. At present more and more people are concerned about the effects of pesticide residues on human health and on the environment.

Compared to chemical treatment, nonchemical methods are safer to man and are generally effective for longer periods of time. One example of nonchemical pest control methods is biological treatment [13-17], including microbial control with pathogens, as disease can be important natural controls of some pests. Insects, like humans and other animals, can be infected by disease-causing organisms such as bacteria, viruses, and fungi.

People also use natural enemy to control pest or regulate it to densities below the threshold for economic damage. Often with augmentation or release, the natural enemy is applied like a pesticide after the pest has reached or exceeded the economic threshold. There are many literatures concerning natural enemy for pest control [18-25].

Many kinds of predator-prey models have been studied extensively $[1,2,6,20,26-28]$. In the natural world there are many species whose individual members have a life history that takes them through two stages: immature and mature. 
In particular, we have in mind mammalian populations and some amphibious animals, which exhibit these two stages. In recent years, stage-structured models, with or without delays, have been studied by several authors [26-33]. In addition, there are many control methods and results for complex dynamical network model [34-38], from which we can learn for the proof of our main results.

Motivated by $[1,14,24,25,33,34]$, in this paper, we will consider predator-prey models with stage structure in the prey. The prey stands for the pest population and the predator stands for the natural enemy population. That is, we call the pest and natural enemy as prey and predator, respectively. Here, the pest population will be controlled by releasing natural enemy and infective pests together. The infective pests can be cultivated in the laboratory and the natural enemy can be migrated from other regions. Once the susceptible pest meets with the infective pest, there is a chance to be infected. The infective pests have more possibility of death due to the disease and have less damage to the crops and environment. In fact, there is such example: salt cedar leaf beetle is a pest, and it is hatched from eggs. We call the egg stage as immature pest, and mature pest after it is hatched. In view of its eggshell, pathogens may not be effective against pest eggs. That is, the disease only attacks the mature susceptible pest. Birds are the natural enemy of the beetle, and we call them predator.

The organization of this paper is as follows. In the next section, the main biological assumptions on which the models rely are formulated and the models are constructed. In Section 3, to prove our main results we give several definitions, notations, and lemmas. In Section 4, we analyze the first case and determine the sufficient conditions for the global attractivity of the susceptible pest-eradication periodic solution and permanence of the system (5). In Section 5, we analyze the second case by similar method and obtain the sufficient conditions for the global attractivity of the susceptible pest-eradication periodic solution and permanence of the system (6). In the last section, a brief discussion and some possible future work for pest management are provided.

\section{Model Formulation}

We assume that the life time of the prey population has two stages, immature stage and mature stage. $x(t)$ represents the density of the immature prey (pest) population. $S(t), I(t)$ represent the densities of the susceptible and infective mature prey (pest) population, respectively. The predator population has only one life time stage. $y(t)$ is the density of the predator (natural enemy) population. And the following assumptions hold.

$\left(H_{1}\right)$ We suppose that the infective prey can neither produce offspring nor attack crops due to the disease, and only the susceptible prey can reproduce. At any time $t>0$, birth into the immature prey population is proportional to the existing susceptible mature prey population with proportionality $r$. The immature prey population will transfer to the mature prey class after its birth with a maturity period of $\tau$. The term $r e^{-d_{1} \tau} S(t-\tau)$ represents the immature prey that were born at time $t-\tau$ (i.e., $r S(t-\tau)$ ) and still survive at time $t$ (with the immature prey death rate $d_{1}$ ), and therefore represent the transformation from immature prey to mature prey.

$\left(H_{2}\right)$ The immature prey population has the natural death rate $d_{1}$. The parameters $d_{2}, d_{3}$ are the death rate for the susceptible and infective mature prey, respectively. The predator population has the natural death rate $d_{4}$.

$\left(\mathrm{H}_{3}\right)$ We also suppose the natural enemy only capture the susceptible mature prey population, since the immature and infective prey population are hidden in the sanctuary, and the predation functional response is type Holling II, $\delta(0<\delta<1)$ is the conversion rate for predation. The mature prey is divided into two classes, susceptible and infective. The incidence rate is classic bilinear $\beta S(t) I(t)$, and $\beta$ is the contact number per unit time for every infective prey with susceptible prey.

$\left(H_{4}\right)$ We assume $\tau_{n}(n=1,2, \ldots)$ and $\lambda_{m}(m=1,2, \ldots)$ are impulsive point series at which the infective prey and natural enemies are released, with the releasing amounts $q_{1}$ and $q_{2}$, respectively. According to the above assumptions, we have two different cases as follows.

Case 1. The releases of infective pests are more frequent than releases of natural enemies.

We release the natural enemies impulsively with releasing amount $q_{2}$ at time $\lambda_{m}$. Assume $\lambda_{m+1}-\lambda_{m}=T_{N}$ holds for all $m\left(m \in Z_{+}\right)$, where $T_{N}$ is the period of releasing natural enemies. During the period, $p$ times of the infective pests are released. That is, there exists an integer $p$, such that $0<\tau_{1}<$ $\tau_{2}<\tau_{3} \cdots<\tau_{p}<T_{N}$. For the convenience of calculation and research, we assume that $\tau_{1}=\tau_{2}-\tau_{1}=\tau_{3}-\tau_{2}=\cdots=T_{N}-\tau_{p}$. Accordingly, the mathematical model will be

$$
\begin{gathered}
\frac{d x(t)}{d t}=r S(t)-d_{1} x(t)-r e^{-d_{1} \tau} S(t-\tau), \\
\left.\begin{array}{c}
\frac{d S(t)}{d t}=r e^{-d_{1} \tau} S(t-\tau)-d_{2} S(t)-\alpha S^{2}(t) \\
-\beta S(t) I(t)-\frac{a S(t)}{1+b S(t)} y(t), \quad
\end{array}\right\} \begin{array}{c}
t \neq \lambda_{m}, \\
t \neq \lambda_{m}+\tau_{i},
\end{array} \\
\frac{d I(t)}{d t}=\beta S(t) I(t)-d_{3} I(t), \\
\frac{d y(t)}{d t}=\frac{\delta a S(t)}{1+b S(t)} y(t)-d_{4} y(t), \quad \Delta S(t)=0, \quad \Delta I(t)=q_{1}, \\
\Delta x(t)=0, \quad t=\lambda_{m}+\tau_{i}, \quad i \in P, m \in Z_{+}, \\
\Delta x(t)=0, \quad \Delta S(t)=0, \quad \Delta I(t)=0, \\
\Delta y(t)=q_{2}, \quad t=\lambda_{m}, \quad m \in Z_{+},
\end{gathered}
$$


where we denote $P=\{1,2, \ldots, p\}$ and $P_{1}=\{1,2, \ldots, p-1\}$ throughout the paper.

Case 2. Natural enemies releases are more frequent than the release of the infective pests.

We release the infective pests impulsively with releasing amount $q_{1}$. Assume that $\tau_{n+1}-\tau_{n} \equiv T_{k}$ holds for all $n \in Z_{+}$, where $T_{k}$ is the period of releasing the infective pests. During the period, $p$ times of natural enemy releases are applied. That is, there exists an integer $p$, such that $0<\lambda_{1}<\lambda_{2}<$ $\lambda_{3} \cdots<\lambda_{p}<T_{k}$. For the simplicity of calculation and research, we assume that $\lambda_{1}=\lambda_{2}-\lambda_{1}=\lambda_{3}-\lambda_{2}=$ $\cdots=T_{k}-\lambda_{p}$. Accordingly, the mathematical model will be

$$
\begin{gathered}
\frac{d x(t)}{d t}=r S(t)-d_{1} x(t)-r e^{-d_{1} \tau} S(t-\tau), \\
\left.\begin{array}{c}
\frac{d S(t)}{d t}=r e^{-d_{1} \tau} S(t-\tau)-d_{2} S(t)-\alpha S^{2}(t) \\
-\beta S(t) I(t)-\frac{a S(t)}{1+b S(t)} y(t), \\
\frac{d I(t)}{d t}=\beta S(t) I(t)-d_{3} I(t), \\
\frac{d y(t)}{d t}=\frac{\delta a S(t)}{1+b S(t)} y(t)-d_{4} y(t),
\end{array}\right\} \begin{array}{c}
t \neq \tau_{n}, \\
t \neq \tau_{n}, \lambda_{i},
\end{array} \\
\Delta x(t)=0, \quad \Delta S(t)=0, \quad \Delta I(t)=0, \\
\Delta y(t)=q_{2}, \quad t=\tau_{n}+\lambda_{i}, \quad i \in P, n \in Z_{+}, \\
\Delta x(t)=0, \quad \Delta S(t)=0, \quad \Delta I(t)=q_{1}, \\
\Delta y(t)=0, \quad t=\tau_{n}, \quad n \in Z_{+} .
\end{gathered}
$$

$\left(H_{5}\right)$ For ecological reasons, we always assume that the initial values $\Phi=\left(\phi_{1}, \phi_{2}, \phi_{3}, \phi_{4}\right)$ for system (1) and (2) satisfy

$$
\phi_{i} \in C\left([-\tau, 0], R_{+}^{4}\right), \quad \phi_{i}(0)>0, \quad i=1,2,3,4,
$$

where $R_{+}^{4}=\{(x, S, I, y): x \geq 0, S \geq 0, I \geq 0, y \geq 0\}$. For continuity of initial conditions, we require

$$
\phi_{1}(0)=\int_{-\tau}^{0} r \phi_{2}(s) e^{d_{1} s} d s
$$

where $\phi_{1}(0)$ represents the accumulated survivors of those prey members who were born between time $-\tau$ and 0 .

For the above two models, note that $x(t)$ will not have impulsive perturbation, and it is not included in the other equations of system (1) and (2), so we only need to consider the following subsystems:

$$
\left.\begin{array}{c}
\frac{d S(t)}{d t}=r e^{-d_{1} \tau} S(t-\tau)-d_{2} S(t)-\alpha S^{2}(t) \\
-\beta S(t) I(t)-\frac{a S(t)}{1+b S(t)} y(t), \\
\frac{d I(t)}{d t}=\beta S(t) I(t)-d_{3} I(t), \\
\frac{d y(t)}{d t}=\frac{\delta a S(t)}{1+b S(t)} y(t)-d_{4} y(t),
\end{array}\right\} \begin{gathered}
t \neq \lambda_{m}, \\
t \neq \lambda_{m}+\tau_{i}, \\
\Delta S(t)=0, \quad \Delta I(t)=q_{1}, \\
\Delta y(t)=0, \quad t=\lambda_{m}+\tau_{i}, i \in P, m \in Z_{+}, \\
\Delta S(t)=0, \quad \Delta I(t)=0, \\
\Delta y(t)=q_{2}, \quad t=\lambda_{m}, \quad m \in Z_{+},
\end{gathered}
$$

$$
\left.\begin{array}{c}
\frac{d S(t)}{d t}=r e^{-d_{1} \tau} S(t-\tau)-d_{2} S(t)-\alpha S^{2}(t) \\
-\beta S(t) I(t)-\frac{a S(t)}{1+b S(t)} y(t), \\
\frac{d I(t)}{d t}=\beta S(t) I(t)-d_{3} I(t), \\
\frac{d y(t)}{d t}=\frac{\delta a S(t)}{1+b S(t)} y(t)-d_{4} y(t),
\end{array}\right\} \begin{gathered}
t \neq \tau_{n}, \\
t \neq \tau_{n}+\lambda_{i}, \\
\Delta S(t)=0, \quad \Delta I(t)=0, \\
\Delta y(t)=q_{2}, \quad t=\tau_{n}+\lambda_{i}, \quad i \in P, n \in Z_{+}, \\
\Delta S(t)=0, \quad \Delta I(t)=q_{1}, \\
\Delta y(t)=0, \quad t=\tau_{n}, n \in Z_{+} .
\end{gathered}
$$

Accordingly, the initial condition of systems (5) and (6) becomes

$$
\phi_{i} \in C\left([-\tau, 0], R_{+}^{3}\right), \quad \phi_{i}(0)>0, \quad i=1,2,3 .
$$

\section{Preliminary}

We give some definitions and lemmas which will be useful for stating and proving our main results.

Definition 1. System (5) and (6) are said to be permanent if there are constants $m, M>0$ (independent of the initial values) and a finite time $T_{0}$ such that for all solution $(S(t), I(t), y(t))$ with initial conditions (7), $m \leq S(t) \leq$ $M, m \leq I(t) \leq M, m \leq y(t) \leq M$ hold for all $t \geq T_{0}$. Here $T_{0}$ may depend on the initial values.

Lemma 2 (see [39]). Consider the following equation:

$$
\frac{d x}{d t}=a x(t-\tau)-b x(t)-c x^{2}(t)
$$


where $a, b, c, \tau$ are positive constants, and $x(t)>0$ for $-\tau \leq t \leq$ 0 , then one has the following.

(i) If $a>b$, then $\lim _{t \rightarrow \infty} x(t)=(a-b) / c$.

(ii) If $a \leq b$, then $\lim _{t \rightarrow \infty} x(t)=0$.

Lemma 3 (see [40]). Let $V: R_{+} \times R^{n} \rightarrow R$ and $V \in V_{0}$. Assume that

$$
\begin{gathered}
D^{+} V(t, X) \leq g(t, V(t, X)), \quad t \neq k \tau, \\
V\left(t, X\left(t^{+}\right)\right) \leq \Psi_{n}(V(t, X(t))), \quad t=k \tau,
\end{gathered}
$$

where $g: R_{+} \times R_{+}^{n} \rightarrow R^{n}$ is continuous in $(k \tau,(k+1) \tau] \times R_{+}^{n}$ and for each $v \in R_{+}^{n}, n \in Z_{+}$

$$
\lim _{(t, y) \rightarrow\left(k \tau^{+}, v\right)} g(t, y)=g\left(k \tau^{+}, v\right)
$$

exists and is finite. $g(t, U)$ is quasi-monotone nondecreasing in $U$ and $\Psi_{n}: R_{+}^{n} \rightarrow R_{+}^{n}$ is nondecreasing. Let $R(t)=R\left(t, 0, U_{0}\right)$ be the maximal solution of the scalar impulsive differential equation

$$
\begin{gathered}
U^{\prime}(t)=g(t, U), \quad t \neq k \tau, \\
U\left(t^{+}\right)=\Psi_{n}(U(t)), \quad t=k \tau, \\
U\left(0^{+}\right)=U_{0}>0,
\end{gathered}
$$

defined on $[0, \infty)$. Then $V\left(0^{+}, X_{0}\right) \leq U_{0}$ implies that $V(t, X(t))$ $\leq R(t), t \geq 0$, where $X(t)$ is any solution of system (1) existing on $[0, \infty)$.

Note that if one has some smoothness conditions of $g$ to guarantee the existence and uniqueness of solutions for (11), then $R(t)$ is exactly the unique solution of (11).

Lemma 4 (see [1]). System

$$
\begin{gathered}
z^{\prime}(t)=-d z(t), \quad t \neq n T, \\
\Delta z(t)=\theta, \quad t=n T,
\end{gathered}
$$

has a positive periodic solution $z^{*}(t)$, and for every solution $z(t)$ of system (12) with positive initial value $z\left(0^{+}\right)$, one has $\mid z(t)-$ $z^{*}(t) \mid \rightarrow 0$ as $t \rightarrow+\infty$, where $z^{*}(t)=\theta e^{-d(t-n T)} /\left(1-e^{-d T}\right)$ for $t \in(n T,(n+1) T]$, and $z^{*}\left(0^{+}\right)=\theta /\left(1-e^{-d T}\right)$.

Lemma 5. There exists a positive constant $M$ such that $S(t) \leq$ $M, I(t) \leq M, y(t) \leq M$, for each solution $(S(t), I(t), y(t))$ of system (5) with positive initial values (7), where $t$ is large enough.

Proof. Define a function $V$ such that

$$
V(t)=\delta S(t)+\delta I(t)+y(t) .
$$

By simple computation, we see that when $t \neq \lambda_{m}, t \neq \lambda_{m}+\tau_{i}$,

$$
\begin{aligned}
\left.D^{+} V\right|_{(5)} & +d V \\
= & \delta r e^{-d_{1} \tau} S(t-\tau)+\delta\left(d-d_{1}\right) S(t) \\
& +\delta\left(d-d_{1}\right) I(t)+\delta\left(d-d_{1}\right) y(t)-\delta a S^{2}(t) \\
\leq & \delta r e^{-d_{1} \tau} S(t-\tau)-\delta a S^{2}(t)
\end{aligned}
$$

Since the right-hand side of the above inequality is a quadratic with negative quadratic coefficient, it is bounded from above for all $(S(t), I(t), y(t)) \in R_{+}^{3}$. Hence there exists a positive constant $M_{0}=r e^{-d_{1} \tau} / a$, such that

$$
\left.D^{+} V\right|_{(5)} \leq-d V(t)+M_{0}, \quad \text { for } t \neq \lambda_{m}, \quad t \neq \lambda_{m}+\tau_{i}
$$

Obviously, we know

$$
\begin{aligned}
& V\left(t^{+}\right)=V(t)+q_{1}, \quad \text { for } t=\lambda_{m}+\tau_{i}, i \in P \\
& V\left(t^{+}\right)=V(t)+q_{2}, \quad \text { for } t=\lambda_{m}, \quad m \in Z_{+} .
\end{aligned}
$$

Consider system

$$
\begin{gathered}
D^{+} V(t) \leq-d V(t)+M_{0}, \quad t \neq \lambda_{m}, t \neq \lambda_{m}+\tau_{i} \\
V\left(t^{+}\right) \leq V(t)+q, \quad t=\lambda_{m}, \text { or } t=\lambda_{m}+\tau_{i},
\end{gathered}
$$

where $d=\min \left\{d_{2}, d_{3}, d_{4}\right\}, q=\max \left\{q_{1}, q_{2}\right\}$.

According to Lemmas 3 and 4, we derive

$$
\begin{aligned}
V(t) \leq & \left(V\left(0^{+}\right)-\frac{M_{0}}{d}-\frac{q}{1-e^{-d T_{N}}}\right) e^{-d t} \\
& +\frac{M_{0}}{d}+\frac{q e^{-d\left(t-\lambda_{m}\right)}}{1-e^{-d T_{N}}} \\
\longrightarrow & \frac{M_{0}}{d}+\frac{q}{1-e^{d T_{N}}}, \quad \text { as } t \longrightarrow \infty .
\end{aligned}
$$

Consequently, by the definition of $V(t)$ we obtain that each solution of (5) with positive initial values is uniformly ultimately bounded above. This completes the proof.

Remark 6. For system (6) with initial value (7), we have similar result as Lemma 5.

\section{Analysis of System (5)}

In this section, we determine the global attractive condition for the susceptible pest-eradication periodic solution and permanence of system (5).

If $S(t) \equiv 0$ for all $t \geq 0$, then we get the following subsystem of system (5):

$$
\begin{gathered}
\left.\begin{array}{c}
\frac{d I(t)}{d t}=-d_{3} I(t), \\
\frac{d y(t)}{d t}=-d_{4} y(t),
\end{array}\right\} t \neq \lambda_{m}, \quad t \neq \lambda_{m}+\tau_{i}, \\
\Delta I(t)=q_{1}, \quad t=\lambda_{m}+\tau_{i}, \quad i \in P, \\
\Delta y(t)=q_{2}, \quad t=\lambda_{m}, \quad m \in Z_{+} .
\end{gathered}
$$


By Lemma 4, system (19) has a unique positive periodic solution as follows:

$$
I^{*}(t)=\left\{\begin{array}{c}
I^{*} e^{-d_{3}\left(t-\lambda_{m}\right)}, \quad t \in\left(\lambda_{m}, \lambda_{m}+\tau_{1}\right], \\
I^{*} e^{-d_{3}\left(t-\lambda_{m}\right)}+q_{1} \sum_{j=1}^{j=i} e^{-d_{3}\left(t-\lambda_{m}-\tau_{j}\right)}, \\
t \in\left(\lambda_{m}+\tau_{i}, \lambda_{m}+\tau_{i+1}\right], i \in P_{1}, \\
\vdots \\
I^{*} e^{-d_{3}\left(t-\lambda_{m}\right)}+q_{1} \sum_{j=1}^{j=p} e^{-d_{3}\left(t-\lambda_{m}-\tau_{j}\right)}, \\
t \in\left(\lambda_{m}+\tau_{p}, \lambda_{m+1}\right],
\end{array}\right.
$$

where

$$
I^{*}=\frac{q_{1} \sum_{j=1}^{j=p} e^{-d_{3}\left(T_{N}-\tau_{j}\right)}}{1-e^{-d_{3} T_{N}}}, \quad y^{*}=\frac{q_{2}}{1-e^{-d_{4} T_{N}}} .
$$

Thus, we get the presentation of the susceptible pest-eradication periodic solution of system $(5)$ as $\left(0, I^{*}(t), y^{*}(t)\right)$. And we have the following results about this periodic solution.

Theorem 7. The susceptible pest-eradication periodic solution of system (5) is globally attractive provided that

$$
r e^{-d_{1} \tau}<d_{2}+\beta I^{*} e^{-d_{3} \tau_{1}}+\frac{a q_{2}}{(1+b M)\left(e^{d_{4} T_{N}}-1\right)} .
$$

Proof. Since (22) holds, we can choose a sufficiently small $\epsilon>$ 0 such that

$$
\begin{aligned}
r e^{-d_{1} \tau}< & d_{2}+\beta\left(I^{*} e^{-d_{3} \tau_{1}}-\epsilon\right) \\
& +\frac{a\left(\left(q_{2} e^{-d_{4} T_{N}} /\left(1-e^{-d_{4} T_{N}}\right)\right)-\epsilon\right)}{1+b M} .
\end{aligned}
$$

Let $(S(t), I(t), y(t))$ be any solution of system (5) with positive initial values. From system (5), we have

$$
\begin{gathered}
\left.\begin{array}{c}
\frac{d I(t)}{d t} \geq-d_{3} I(t), \\
\frac{d y(t)}{d t} \geq-d_{4} y(t),
\end{array}\right\} t \neq \lambda_{m}, \quad t \neq \lambda_{m}+\tau_{i}, \\
\Delta I(t)=q_{1}, \quad t=\lambda_{m}+\tau_{i}, \quad i \in P, \\
\Delta y(t)=q_{2}, \quad t=\lambda_{m}, \quad m \in Z_{+} .
\end{gathered}
$$

According to Lemmas 3 and 4, for the above $\epsilon$, there exists $n_{1} \in Z_{+}$, such that

$$
\begin{gathered}
I(t) \geq I^{*}(t)-\epsilon \geq I^{*} e^{-d_{3} \tau_{1}}-\epsilon \triangleq \eta_{1}, \\
y(t) \geq y^{*}(t)-\epsilon \geq \frac{q_{2} e^{-d_{4} T_{N}}}{1-e^{-d_{4} T_{N}}}-\epsilon \triangleq \eta_{2}
\end{gathered}
$$

hold for all $t>n_{1} T_{N}$.
Thus, for $t>n_{1} T_{N}+\tau$, we have

$$
\begin{aligned}
\dot{S}(t)= & r e^{-d_{1} \tau} S(t-\tau)-d_{2} S(t) \\
& -\alpha S^{2}(t)-\beta S(t) I(t)-\frac{a S(t)}{1+b S(t)} y(t), \\
\leq & r e^{-d_{1} \tau} S(t-\tau) \\
& -\left[d_{2}+\beta \eta_{1}+\frac{a \eta_{2}}{1+b M}\right] S(t)-\alpha S^{2}(t) .
\end{aligned}
$$

Consider the following comparison equation:

$$
\begin{aligned}
\dot{z}_{1}(t)= & r e^{-d_{1} \tau} z_{1}(t-\tau) \\
& -\left[d_{2}+\beta \eta_{1}+\frac{a \eta_{2}}{1+b M}\right] z_{1}(t)-\alpha z_{1}^{2}(t) .
\end{aligned}
$$

By Lemma 2, we have $\lim _{t \rightarrow \infty} z_{1}(t)=0$. And by Lemma 3, we have $S(t)<z_{1}(t)$, for $t>n_{1} T_{N}+\tau$ from which we get $\lim _{t \rightarrow \infty} S(t)=0$. Therefore, for any positive constant $\epsilon_{1}$ small enough $\left(0<\epsilon_{1}<\min \left(\left(d_{3} / \beta\right),\left(d_{4} /\left(\delta a-b d_{4}\right)\right)\right)\right)$, there exists an integer $n_{2}\left(n_{2} T_{N}>n_{1} T_{N}+\tau\right)$ such that $0<S(t)<\epsilon_{1}$ for all $t>n_{2} T_{N}$.

From system (5), for all $t>n_{2} T_{N}$ we have,

$$
\begin{aligned}
& \left.\begin{array}{c}
\frac{d I(t)}{d t} \leq\left(\beta \epsilon_{1}-d_{3}\right) I(t), \\
\frac{d y(t)}{d t} \leq\left(\frac{\delta a \epsilon_{1}}{1+b \epsilon_{1}}-d_{4}\right) y(t),
\end{array}\right\} t \neq \lambda_{m}, t \neq \lambda_{m}+\tau_{i}, \\
& \Delta I(t)=q_{1}, \quad t=\lambda_{m}+\tau_{i}, \quad i \in P, \\
& \Delta y(t)=q_{2}, \quad t=\lambda_{m}, \quad m \in Z_{+} .
\end{aligned}
$$

From Lemmas 3 and 4 , for any sufficiently small positive $\epsilon_{2}$, there exists an integer $n_{3}\left(n_{3}>n_{2}\right)$ such that

$$
\begin{aligned}
& I(t) \leq \omega_{1}^{*}(t)+\epsilon_{2}, \\
& y(t) \leq \omega_{2}^{*}(t)+\epsilon_{2}
\end{aligned}
$$

for all $t>n_{3} T_{N}$, where

$$
\begin{gathered}
\omega_{1}^{*}(t)=\left\{\begin{array}{l}
\omega_{1}^{*} e^{\left(\beta \epsilon_{1}-d_{3}\right)\left(t-\lambda_{m}\right)}, \quad t \in\left(\lambda_{m}, \lambda_{m}+\tau_{1}\right], \\
\omega_{1}^{*} e^{\left(\beta \epsilon_{1}-d_{3}\right)\left(t-\lambda_{m}\right)}+q_{1} \sum_{j=1}^{j=i} e^{\left(\beta \epsilon_{1}-d_{3}\right)\left(t-\lambda_{m}-\tau_{j}\right)}, \\
\quad t \in\left(\lambda_{m}+\tau_{i}, \lambda_{m}+\tau_{i+1}\right], i \in P_{1}, \\
\omega_{2}^{*}(t)= \\
\omega_{1}^{*} e^{\left(\beta \epsilon_{1}-d_{3}\right)\left(t-\lambda_{m}\right)}+q_{1} \sum_{j=1}^{j=p} e^{\left(\left(\beta \epsilon_{1}-d_{3}\right)\left(t-\lambda_{m}-\tau_{j}\right)\right.} \\
\quad t \in\left(\lambda_{m}+\tau_{p}, \lambda_{m+1}\right], \\
\omega_{1}^{*}=\frac{q_{1} \sum_{j=1}^{j=p} e^{\left(\beta \epsilon_{1}-d_{3}\right)\left(T_{N}-\tau_{j}\right)}}{1-e^{-d_{3} T_{N}}}, \\
\omega_{2}^{*}=\frac{q_{2}}{1-e^{\left(\left(\delta a \epsilon_{1} /\left(1+\epsilon_{1}\right)\right)-d_{4}\right) T_{N}}}
\end{array}\right.
\end{gathered}
$$


are the solutions of the following comparison system:

$$
\begin{aligned}
& \left.\begin{array}{c}
\frac{d \omega_{1}(t)}{d t}=\left(\beta \epsilon_{1}-d_{3}\right) \omega_{1}(t), \\
\frac{d \omega_{2}(t)}{d t}=\left(\frac{\delta a \epsilon_{1}}{1+b \epsilon_{1}}-d_{4}\right) \omega_{2}(t),
\end{array}\right\} \begin{array}{c}
t \neq \lambda_{m} \\
t \neq \lambda_{m}+\tau_{i}
\end{array} \\
& \Delta \omega_{1}(t)=q_{1}, \quad t=\lambda_{m}+\tau_{i}, \quad i \in P, \\
& \Delta \omega_{2}(t)=q_{2}, \quad t=\lambda_{m}, \quad m \in Z_{+}, \\
& \omega_{1}\left(n_{3} T_{N}^{+}\right)=I\left(n_{3} T_{N}^{+}\right)>0, \\
& \omega_{2}\left(n_{3} T_{N}^{+}\right)=y\left(n_{3} T_{N}^{+}\right)>0 .
\end{aligned}
$$

Since $\epsilon, \epsilon_{1}, \epsilon_{2}$ can be small enough, we have $\omega_{1}^{*}(t) \rightarrow I^{*}(t)$, $\omega_{2}^{*}(t) \rightarrow y^{*}(t)$ as $\epsilon, \epsilon_{1}, \epsilon_{2} \rightarrow 0$. From (25) and (29), we deduce that $S(t) \rightarrow 0, I(t) \rightarrow I^{*}(t), y(t) \rightarrow y^{*}(t)$ as $t \rightarrow \infty$. Therefore, $\left(0, I^{*}(t), y^{*}(t)\right)$ is globally attractive. This completes the proof.

Corollary 8. (1) If $q_{1}=0$, then the condition (22) of Theorem 7 becomes

$$
q_{2}>\frac{\left(r e^{-d_{1} \tau}-d_{2}\right)(1+b M)\left(e^{d_{4} T_{N}}-1\right)}{a} \triangleq q_{2 *}
$$

which means that if only natural enemies are released impulsively, then the release amount must be larger than $q_{2 *}$ to ensure the eradication of the susceptible pest.

(2) If $q_{2}=0$, then the condition (22) of Theorem 7 becomes

$$
q_{1}>\frac{\left(r e^{-d_{1} \tau}-d_{2}\right)\left(1-e^{-d_{3} T_{N}}\right)}{\beta \sum_{j=1}^{j=p} e^{-d_{3}\left(T_{N}-\left(\tau_{j}-\tau_{1}\right)\right)}} \triangleq q_{1 *}
$$

which means that if only infective pests are released impulsively, then the release amount must be larger than $q_{1 *}$ to ensure the eradication of the susceptible pest.

Through Theorem 7 and Corollary 8, we can get the sufficient conditions for global attractivity of the susceptible pests-eradication periodic solution. That is, the susceptible pest population is eradicated totally. But in practice, from the view point of keeping ecosystem balance and preserving biological resources, it is not necessary to eradicate the susceptible pest population completely. In fact we hope the susceptible pests and natural enemies can coexist, and at the same time the susceptible pests do not cause immense economic loss. Thus, it is meaningful to study the permanence of system (5).
Theorem 9. The system (5) is permanent provided that

$$
r e^{-d_{1} \tau}>d_{2}+\beta q_{1} \sum_{j=1}^{p} \frac{e^{-d_{3}\left(\tau_{p}-\tau_{j}\right)}}{1-e^{-d_{3} T_{N}}}+\frac{a q_{2}}{1-e^{-d_{4} T_{N}}} .
$$

Proof. Since (34) holds, we can choose sufficiently small positive number $\epsilon, \eta_{4}\left(0<\eta_{4}<\min \left(\left(d_{3} / \beta\right),\left(d_{4} /(\delta a-\right.\right.\right.$ $\left.\left.\left.b d_{4}\right)\right)\right)$ ) such that

$$
\begin{aligned}
\xi= & r e^{-d_{1} \tau}-d_{2}-\alpha \eta_{4}-\beta\left(\frac{q_{1} \sum_{j=1}^{j=p} e^{\left(\beta \eta_{4}-d_{3}\right)\left(\tau_{p}-\tau_{j}\right)}}{1-e^{\left(\beta \eta_{4}-d_{3}\right) T_{N}}}+\epsilon\right) \\
& -a\left(\frac{q_{2}}{1-e^{\left(\left(\delta a \eta_{4} /\left(1+b \eta_{4}\right)\right)-d_{4}\right) T_{N}}}+\epsilon\right)>0 .
\end{aligned}
$$

Suppose $X(t)=(S(t), I(t), y(t))$ is a solution of system (5) with initial values $X\left(0^{+}\right)>0$. By Lemma 5, there exists a positive constant $M$ such that $S(t)<M, I(t)<M, y(t)<M$ for all $t$ large enough. From (25), we know that $I(t), y(t)$ are positive lower bound. Thus we only need to find $\eta_{3}>0$ such that $S(t)>\eta_{3}$ for all $t$ large enough.

We claim that for any $t_{0}>0$, it is impossible that $S(t)<\eta_{4}$ for all $t \geq t_{0}$. Suppose that the claim is not true. Then there is a $t_{0}>0$ such that $S(t)<\eta_{4}$ for all $t \geq t_{0}$.

Then, from system (5) we have

$$
\left.\begin{array}{c}
\dot{I}(t)=\beta S(t) I(t)-d_{3} I(t) \\
\leq\left(\beta \eta_{4}-d_{3}\right) I(t), \\
\dot{y}(t)=\frac{\delta a S(t)}{1+b S(t)} y(t)-d_{4} y(t) \\
\leq\left(\frac{\delta a \eta_{4}}{1+b \eta_{4}}-d_{4}\right) y(t),
\end{array}\right\} \begin{gathered}
t \neq \lambda_{m}, \\
t \neq \lambda_{m}+\tau_{i}, \\
\Delta I(t)=q_{1}, \quad t=\lambda_{m}+\tau_{i}, \\
\Delta y(t)=q_{2}, \quad t=\lambda_{m} .
\end{gathered}
$$

From Lemmas 3 and 4, for the above selected $\epsilon$ in (35), there exists a time $T_{1}>t_{0}+\tau$ such that for all $t \geq T_{1}$ we have

$$
\begin{aligned}
I(t) & \leq u_{1}^{*}(t)+\epsilon \\
& <\frac{q_{1} \sum_{j=1}^{j=p} e^{\left(\beta \eta_{4}-d_{3}\right)\left(\tau_{p}-\tau_{j}\right)}}{1-e^{\left(\beta \eta_{4}-d_{3}\right) T_{N}}}+\epsilon \triangleq \xi_{1}, \\
y(t) & \leq u_{2}^{*}(t)+\epsilon \\
& <\frac{q_{2}}{1-e^{\left(\left(\delta a \eta_{4} /\left(1+b \eta_{4}\right)\right)-d_{4}\right) T_{N}}}+\epsilon \triangleq \xi_{2},
\end{aligned}
$$


where $u_{1}^{*}(t), u_{2}^{*}(t)$ are the solutions of the following comparison system:

$$
\begin{aligned}
& \left.\begin{array}{c}
\dot{u}_{1}(t)=\left(\beta \eta_{4}-d_{3}\right) u_{1}(t), \\
\dot{u}_{2}(t)=\left(\frac{\delta a \eta_{4}}{1+b \eta_{4}}-d_{4}\right) u_{2}(t),
\end{array}\right\} \begin{array}{c}
t \neq \lambda_{m}, \\
t \neq \lambda_{m}+\tau_{i},
\end{array} \\
& \Delta u_{1}(t)=q_{1}, \quad t=\lambda_{m}+\tau_{i}, \\
& \Delta u_{2}(t)=q_{2}, \quad t=\lambda_{m}, \\
& u_{1}\left(T_{1}^{+}\right)=I\left(T_{1}^{+}\right)>0 \text {, } \\
& u_{2}\left(T_{1}^{+}\right)=y\left(T_{1}^{+}\right)>0 \text {, } \\
& u_{1}^{*}(t)=\left\{\begin{array}{rr}
u_{1}^{*} e^{\left(\beta \eta_{4}-d_{3}\right)\left(t-\lambda_{m}\right)}, & t \in\left(\lambda_{m}, \lambda_{m}+\tau_{1}\right], \\
u_{1}^{*} e^{\left(\beta \eta_{4}-d_{3}\right)\left(t-\lambda_{m}\right)}+q_{1} \sum_{j=1}^{j=i} e^{\left(\beta \eta_{4}-d_{3}\right)\left(t-\lambda_{m}-\tau_{j}\right)}, \\
t \in\left(\lambda_{m}+\tau_{i}, \lambda_{m}+\tau_{i+1}\right], \\
\vdots \\
u_{1}^{*} e^{\left(\beta \eta_{4}-d_{3}\right)\left(t-\lambda_{m}\right)}+q_{1} \sum_{j=1}^{j=p} e^{\left(\beta \eta_{4}-d_{3}\right)\left(t-\lambda_{m}-\tau_{j}\right)}, \\
t \in\left(\lambda_{m}+\tau_{p}, \lambda_{m+1}\right],
\end{array}\right. \\
& u_{2}^{*}(t)=u_{2}^{*} e^{\left(\left(\delta a \eta_{4} /\left(1+b \eta_{4}\right)\right)-d_{4}\right)\left(t-\lambda_{m}\right)}, \quad t \in\left(\lambda_{m}, \lambda_{m+1}\right], \\
& u_{1}^{*}=\frac{q_{1} \sum_{j=1}^{j=p} e^{\left(\beta \eta_{4}-d_{3}\right)\left(T_{N}-\tau_{j}\right)}}{1-e^{\left(\beta \eta_{4}-d_{3}\right) T_{N}}}, \\
& u_{2}^{*}=\frac{q_{2}}{1-e^{\left(\left(\delta a \eta_{4} /\left(1+b \eta_{4}\right)\right)-d_{4}\right) T_{N}}} .
\end{aligned}
$$

Note that the first equation of system (5) can be rewritten as

$$
\begin{aligned}
\dot{S}(t)= & r e^{-d_{1} \tau} S(t-\tau)-d_{2} S(t)-\alpha S^{2}(t) \\
& -\beta S(t) I(t)-\frac{a S(t)}{1+b S(t)} y(t), \\
= & {\left[r e^{-d_{1} \tau}-d_{2}-\alpha S(t)-\beta I(t)-\frac{a y(t)}{1+b S(t)}\right] S(t) } \\
& -r e^{-d_{1} \tau} \frac{d}{d t}\left(\int_{t-\tau}^{t} S(u) d u\right) .
\end{aligned}
$$

In the following we define a Liapunov functional as

$$
U(t)=S(t)+r e^{-d_{1} \tau} \int_{t-\tau}^{t} S(u) d u .
$$

Then the derivative of $U(t)$ with respect to the solution of system (5) is

$$
\begin{aligned}
\dot{U}(t)= & r e^{-d_{1} \tau} S(t)-d_{2} S(t)-\alpha S^{2}(t) \\
& -\beta S(t) I(t)-\frac{a S(t)}{1+b S(t)} y(t), \\
= & {\left[r e^{-d_{1} \tau}-d_{2}-\alpha S(t)-\beta I(t)-\frac{a y(t)}{1+b S(t)}\right] S(t) . }
\end{aligned}
$$

From (37) and (41) we have

$$
\begin{aligned}
& \dot{U}(t)= {\left[r e^{-d_{1} \tau}-d_{2}-\alpha S(t)-\beta I(t)-\frac{a y(t)}{1+b S(t)}\right] S(t) } \\
& \geq\left[r e^{-d_{1} \tau}-d_{2}-\alpha S(t)-\beta\left(u_{1}^{*}(t)+\epsilon\right)\right. \\
&\left.-a\left(u_{2}^{*}(t)+\epsilon\right)\right] S(t) .
\end{aligned}
$$

Let $\min _{t \in\left[T_{1}, T_{1}+\tau\right]} S(t)=S_{0}>0$.

We will show that $S(t) \geq S_{0}$ for all $t>T_{1}$. Otherwise there exists a nonnegative constant $T_{2}$ such that $S(t) \geq S_{0}$ for $t \in\left[T_{1}, T_{1}+\tau+T_{2}\right], S\left(T_{1}+\tau+T_{2}\right)=S_{0}$ and $\dot{S}\left(T_{1}+\tau+T_{2}\right) \leq 0$. Thus from the first equation of system (5) and (35) we easily see that

$$
\begin{aligned}
\dot{S}\left(T_{1}+\right. & \left.\tau+T_{2}\right) \\
= & r e^{-d_{1} \tau} S\left(T_{1}+T_{2}\right)-d_{2} S\left(T_{1}+\tau+T_{2}\right) \\
& -\alpha S^{2}\left(T_{1}+\tau+T_{2}\right)-\beta S\left(T_{1}+\tau+T_{2}\right) \\
& \times I\left(T_{1}+\tau+T_{2}\right)-\frac{a S\left(T_{1}+\tau+T_{2}\right)}{1+b S\left(T_{1}+\tau+T_{2}\right)} \\
& \times y\left(T_{1}+\tau+T_{2}\right), \\
\geq & S_{0}\left[r e^{-d_{1} \tau}-d_{2}-\alpha \eta_{4}-\beta\left(u_{1}^{*}(t)+\epsilon\right)\right. \\
& \left.\quad-a\left(u_{2}^{*}(t)+\epsilon\right)\right]>S_{0} \xi>0
\end{aligned}
$$

which is a contradiction. Hence we get that $S(t) \geq S_{0}>0$ for all $t \geq T_{1}$.

From (37) we have for all $t>T_{1}$

$$
\begin{aligned}
\dot{U}(t)= & r e^{-d_{1} \tau} S(t)-d_{2} S(t)-\alpha S^{2}(t) \\
& -\beta S(t) I(t)-\frac{a S(t)}{1+b S(t)} y(t), \\
= & {\left[r e^{-d_{1} \tau}-d_{2}-\alpha S(t)-\beta I(t)-\frac{a y(t)}{1+b S(t)}\right] S(t) } \\
> & S_{0}\left[r e^{-d_{1} \tau}-d_{2}-\alpha \eta_{4}\right. \\
& \left.\quad-\beta\left(u_{1}^{*}(t)+\epsilon\right)-a\left(u_{2}^{*}(t)+\epsilon\right)\right]
\end{aligned}
$$$$
>\mathrm{S}_{0} \xi>0 \text {, }
$$

which implies $U(t) \rightarrow \infty$ as $t \rightarrow \infty$. This is a contradiction to $U(t) \leq M+r \tau M e^{-d_{1} \tau}$. Therefore for any positive constant $t_{0}$ the inequality $S(t)<\eta_{4}$ cannot hold for all $t \geq t_{0}$.

By the claim, we need to consider two cases.

Case 1. $\left(S(t)>\eta_{4}\right)$ for all large $t$, then our aim is obtained.

Case 2. $(S(t))$ oscillates about $\eta_{4}$ for all large $t$. Denote

$$
\eta_{3}=\min \left(\frac{\eta_{4}}{2}, \eta_{4} e^{-\left(d_{2}+\alpha M+\beta \xi_{1}+a \xi_{2}\right) \tau}\right) .
$$


In the following we will show that $S(t) \geq \eta_{\underline{3}}$. Since $S(t)$ oscillates about $\eta_{4}$, there exist positive constants $\bar{t}$ and $\theta$, such that

$$
\begin{gathered}
S(\bar{t})=S(\bar{t}+\theta)=\eta_{4}, \\
S(t)<\eta_{4}, \quad \bar{t}<t<\bar{t}+\theta,
\end{gathered}
$$

where $\bar{t}$ is sufficiently large, and the inequalities (37) hold for $\bar{t}<t<\bar{t}+\theta$. Since $S(t)$ is continuous and bounded, and it is not effected by impulse. So there exists a constant $T_{3}$ (where $0<T_{3}<\tau$ and $T_{3}$ is independent of the choice of $\bar{t}$ ) such that $S(t)>\eta_{4} / 2$ for all $\bar{t} \leq t \leq \bar{t}+T_{3}$.

If $\theta \leq T_{3}$, our aim is obtained.

If $T_{3}<\theta<\tau$, from the first equation of system (5) we have, for $\bar{t} \leq t \leq \bar{t}+\theta$

$$
\begin{aligned}
\dot{S}(t) & \geq\left(-d_{2}-\alpha S(t)-\beta I(t)-a y(t)\right) S(t) \\
& \geq-\left(d_{2}+\alpha M+\beta \xi_{1}+a \xi_{2}\right) S(t),
\end{aligned}
$$

then

$$
S(t) \geq \eta_{4} e^{-\left(d_{2}+\alpha M+\beta \xi_{1}+a \xi_{2}\right) \tau} \quad \text { for } \bar{t} \leq t \leq \bar{t}+\theta \leq \bar{t}+\tau .
$$

It is clear that $S(t) \geq \eta_{3}$ for $\bar{t} \leq t \leq \bar{t}+\theta$.

If $\theta>\tau$, then we have that $S(t) \geq \eta_{3}$ for $\bar{t} \leq t \leq \bar{t}+\tau$. Then, proceeding exactly as the proof for above claim, we see that $S(t) \geq \eta_{3}$ for $\bar{t}+\tau \leq t \leq \bar{t}+\theta$. Since this kind of interval $[\bar{t}, \bar{t}+\theta]$ is arbitrarily chosen (we only need $\bar{t}$ to be large), we can get that $S(t) \geq \eta_{3}$ for all $t$ large enough. In view of our arguments above, the choice of $\eta_{3}$ is independent of the positive solution of system (5) which satisfies that $S(t) \geq \eta_{3}$ for sufficiently large $t$. This completes the proof of the theorem.

Corollary 10. (1) If $q_{1}=0$, then condition (34) of Theorem 9 becomes

$$
q_{2}<\frac{\left(r e^{-d_{1} \tau}-d_{2}\right)\left(1-e^{d_{4} T_{N}}\right)}{a}=q_{2}^{*}
$$

which means that if only natural enemies are released impulsively, and the release amount is less than $q_{2}^{*}$, then system (5) is permanent. That is, the pest and natural enemy will coexist.

(2) If $q_{2}=0$, then condition (34) of Theorem 9 becomes

$$
q_{1}<\frac{r e^{-d_{1} \tau}-d_{2}}{\beta \sum_{j=1}^{p}\left(e^{-d_{3}\left(\tau_{p}-\tau_{j}\right)} /\left(1-e^{-d_{3} T_{N}}\right)\right)}=q_{1}^{*}
$$

which means that if only infective pests are released impulsively, and the release amount is less than $q_{1}^{*}$, then system (5) is permanent. That is, the pest and natural enemy will coexist.

\section{Analysis of System (6)}

In this section we will discuss system (6), the condition for the global attractive of the susceptible pest-eradication periodic solution, and the permanence of system (6) will be obtained.
If $S(t) \equiv 0$ for all $t \geq 0$, then we get the following subsystem of system (6):

$$
\begin{aligned}
& \left.\begin{array}{l}
\dot{I}(t) \geq-d_{3} I(t), \\
\dot{y}(t) \geq-d_{4} y(t),
\end{array}\right\} t \neq \tau_{n}, t \neq \tau_{n}+\lambda_{i}, \\
& \Delta y(t)=q_{2}, \quad t=\tau_{n}+\lambda_{i}, i \in P, \\
& \Delta I(t)=q_{1}, \quad t=\tau_{n}, n \in z_{+} .
\end{aligned}
$$

By Lemma 4, we know that the system (51) has a unique positive periodic solution as follows:

$$
\begin{gathered}
y^{*}(t)=\left\{\begin{array}{l}
y^{*} e^{-d_{4}\left(t-\tau_{n}\right)}, \quad t \in\left(\tau_{n}, \tau_{n}+\lambda_{1}\right], \\
y^{*} e^{-d_{4}\left(t-\tau_{n}\right)}+q_{2} \sum_{j=1}^{j=i} e^{-d_{4}\left(t-\tau_{n}-\lambda_{j}\right)}, \\
\vdots \\
\quad t \in\left(\tau_{n}+\lambda_{i}, \tau_{n}+\lambda_{i+1}\right], \\
y^{*} e^{-d_{4}\left(t-\tau_{n}\right)}+q_{2} \sum_{j=1}^{j=p} e^{-d_{4}\left(t-\tau_{n}-\lambda_{j}\right)}, \\
\quad t \in\left(\tau_{n}+\lambda_{p}, \tau_{n+1}\right],
\end{array}\right. \\
I^{*}(t)=\frac{q^{*} e^{-d_{3}\left(t-\tau_{n}\right)},}{\sum_{j=1}^{j=p} e^{-d_{4}\left(T_{k}-\lambda_{j}\right)}} \quad t \in\left(\tau_{n}, \tau_{n+1}\right], \\
1-e^{-d_{4} T_{k}}, \quad I^{*}=\frac{q_{1}}{1-e^{-d_{3} T_{k}}} .
\end{gathered}
$$

Thus, system (6) has a unique nonnegative periodic solution $\left(0, I^{*}(t), y^{*}(t)\right)$, which is called as the susceptible pesteradication periodic solution. Next, we will discuss the global attractivity of this periodic solution and the permanence of system (6).

Theorem 11. The susceptible pest-eradication periodic solution of system (6) is attractive provided that

$$
r e^{-d_{1} \tau}<d_{2}+\frac{q_{1} \beta}{e^{d_{3} T_{k}}-1}+\frac{a y^{*} e^{-d_{4} \lambda_{1}}}{1+b M} .
$$

Proof. Since (53) holds, we can choose a sufficiently small $\epsilon>$ 0 such that

$$
r e^{-d_{1} \tau}<d_{2}+\beta\left(I^{*}(t)-\epsilon\right)+\frac{a\left(y^{*}(t)-\epsilon\right)}{1+b M} .
$$

Let $(S(t), I(t), y(t))$ be any solution of system (6) with initial values (7). From system (6), we have

$$
\begin{aligned}
& \left.\begin{array}{l}
\dot{I}(t) \geq-d_{3} I(t), \\
\dot{y}(t) \geq-d_{4} y(t),
\end{array}\right\} \quad t \neq \tau_{n}, \quad t \neq \tau_{n}+\lambda_{i}, \\
& \Delta y(t)=q_{2}, \quad t=\tau_{n}+\lambda_{i}, i \in P, \\
& \Delta I(t)=q_{1}, \quad t=\tau_{n}, n \in z_{+} .
\end{aligned}
$$

From Lemmas 3 and 4 , for the above selected $\epsilon$, there exists $n_{1} \in Z_{+}$, such that for all $t>n_{1} T_{k}$ we have

$$
\begin{aligned}
& I(t) \geq I^{*}(t)-\epsilon \geq \frac{q_{1} e^{-d_{3} T_{k}}}{1-e^{-d_{3} T_{k}}}-\epsilon \doteq h_{1}, \\
& y(t) \geq y^{*}(t)-\epsilon \geq y^{*} e^{-d_{4} \lambda_{1}}-\epsilon \doteq h_{2} .
\end{aligned}
$$


Thus, for $t>n_{1} T_{k}+\tau$, we have

$$
\begin{aligned}
\dot{S}(t)= & r e^{-d_{1} \tau} S(t-\tau)-d_{2} S(t)-\alpha S^{2}(t) \\
& -\beta S(t) I(t)-\frac{a S(t)}{1+b S(t)} y(t), \\
\leq & r e^{-d_{1} \tau} S(t-\tau)-\left[d_{2}+\beta h_{1}+\frac{a h_{2}}{1+b M}\right] S(t)-\alpha S^{2}(t) .
\end{aligned}
$$

Consider the following comparison equation:

$$
\begin{aligned}
\dot{z}_{2}(t)= & r e^{-d_{1} \tau} z_{2}(t-\tau) \\
& -\left(d_{2}+\beta h_{1}+\frac{a h_{2}}{1+b M}\right) z_{1}(t)-\alpha z_{2}^{2}(t) .
\end{aligned}
$$

By Lemma 2, we have $\lim _{t \rightarrow \infty} z_{2}(t)=0$ and by Lemma 3, we have $S(t)<z_{1}(t)$, for large $t$, that is $\lim _{t \rightarrow \infty} S(t)=0$. Therefore, for any positive constant $\epsilon_{1}$ small enough $\left(0<\epsilon_{1}<\right.$ $\left.\min \left(d_{3} / \beta, d_{4} /\left(\delta a-b d_{4}\right)\right)\right)$, there exists an integer $n_{2}\left(n_{2} T_{k}>\right.$ $\left.n_{1} T_{k}+\tau\right)$ such that $0<S(t)<\epsilon_{1}$ for all $t>n_{2} T_{k}$.

Thus, from system (6), for all $t>n_{2} T_{k}$ we have

$$
\begin{aligned}
& \left.\begin{array}{c}
\dot{I}(t) \leq\left(\beta \epsilon-d_{3}\right) I(t), \\
\dot{y}(t) \leq\left(\frac{\delta a \epsilon}{1+b \epsilon}-d_{4}\right) y(t),
\end{array}\right\} t \neq \tau_{n}, t \neq \tau_{n}+\lambda_{i}, \\
& \Delta y(t)=q_{2}, \quad t=\tau_{n}+\lambda_{i}, i \in P, \\
& \Delta I(t)=q_{1}, \quad t=\tau_{n}, n \in z_{+} .
\end{aligned}
$$

From Lemmas 3 and 4 , for a sufficiently small $\epsilon_{2}>0$, there exists an integer $n_{3}\left(n_{3}>n_{2}\right)$ such that

$$
\begin{aligned}
& I(t) \leq \vartheta_{1}^{*}(t)+\epsilon_{2}, \\
& y(t) \leq \vartheta_{2}^{*}(t)+\epsilon_{2},
\end{aligned}
$$

for all $t>n_{3} T_{k}$, where

$$
\begin{gathered}
\vartheta_{1}^{*}(t)=\vartheta_{1}^{*} e^{\left(\beta \epsilon-d_{3}\right)\left(t-\tau_{n}\right)}, \quad t \in\left(\tau_{n}, \tau_{n+1}\right], \quad \vartheta_{1}^{*}=\frac{q_{1}}{1-e^{\left(\beta \epsilon-d_{3}\right) T_{k}}}, \\
\vartheta_{2}^{*}(t)= \begin{cases}\vartheta_{2}^{*} e^{\left((\delta a \epsilon /(1+b \epsilon))-d_{4}\right)\left(t-\tau_{n}\right)}, & t \in\left(\tau_{n}, \tau_{n}+\lambda_{1}\right], \\
\vartheta_{2}^{*} e^{\left((\delta a \epsilon /(1+b \epsilon))-d_{4}\right)\left(t-\tau_{n}\right)}+q_{2} \sum_{j=1}^{j=i} e^{\left((\delta a \epsilon /(1+b \epsilon))-d_{4}\right)\left(t-\tau_{n}-\lambda_{j}\right)}, & t \in\left(\tau_{n}+\tau_{i}, \tau_{n}+\lambda_{i+1}\right], i \in P_{1}, \\
\vdots & \\
\vartheta_{2}^{*} e^{\left((\delta a \epsilon /(1+b \epsilon))-d_{4}\right)\left(t-\tau_{n}\right)}+q_{2} \sum_{j=1}^{j=i} e^{\left((\delta a \epsilon /(1+b \epsilon))-d_{4}\right)\left(t-\tau_{n}-\lambda_{j}\right)}, & t \in\left(\tau_{n}+\tau_{p}, \tau_{n+1}\right], \\
\vartheta_{2}^{*}=\frac{q_{2} \sum_{j=1}^{j=p} e^{\left((\delta a \epsilon /(1+b \epsilon))-d_{4}\right)\left(T_{k}-\lambda_{j}\right)}}{1-e^{\left((\delta a \epsilon /(1+b \epsilon))-d_{4}\right) T_{k}}}\end{cases}
\end{gathered}
$$

is the solution of the following comparison system:

$$
\begin{aligned}
& \left.\begin{array}{c}
\dot{\vartheta}_{1}(t)=\left(\beta \epsilon-d_{3}\right) \vartheta_{1}(t), \\
\dot{\vartheta}_{2}(\mathrm{t})=\left(\frac{\delta a \epsilon}{1+b \epsilon}-d_{4}\right) \vartheta_{2}(t),
\end{array}\right\} \begin{array}{c}
t \neq \tau_{n}, \\
t \neq \tau_{n}+\lambda_{i},
\end{array} \\
& \Delta \vartheta_{2}(t)=q_{2}, \quad t=\tau_{n}+\lambda_{i}, i \in P, \\
& \Delta \vartheta_{1}(t)=q_{1}, \quad t=\tau_{n}, n \in z_{+}, \\
& \vartheta_{1}\left(n_{3} T_{k}^{+}\right)=I\left(n_{3} T_{k}^{+}\right), \\
& \vartheta_{2}\left(n_{3} T_{k}^{+}\right)=y\left(n_{3} T_{k}^{+}\right) \text {. }
\end{aligned}
$$

Since $\epsilon, \epsilon_{1}, \epsilon_{2}$ can be small enough, we have $\vartheta_{1}^{*}(t) \rightarrow I^{*}(t)$, $\vartheta_{2}^{*}(t) \rightarrow y^{*}(t)$ as $\epsilon, \epsilon_{1}, \epsilon_{2} \rightarrow 0$. From (56) and (60) we get $I(t) \rightarrow I^{*}(t), y(t) \rightarrow y^{*}(t)$ as $t \rightarrow+\infty$. Together with $S(t) \rightarrow 0$, we obtain that $\left(0, I^{*}(t), y^{*}(t)\right)$ is globally attractive. This completes the proof.
Corollary 12. (1) If $q_{1}=0$, then condition (53) of Theorem 11 becomes

$$
q_{2}>\frac{\left(r e^{-d_{1} \tau}-d_{2}\right)(1+b M)\left(1-e^{-d_{4} T_{k}}\right)}{a \sum_{j=1}^{j=p} e^{-d_{4}\left(T_{k}-\left(\lambda_{j}-\lambda_{1}\right)\right)}} \doteq \overline{q_{2 *}},
$$

which means that if only natural enemies are released impulsively, then the release amount must be larger than $\overline{q_{2 *}}$ to ensure the eradication of the susceptible pest.

(2) If $q_{2}=0$, then condition (53) of Theorem 11 becomes

$$
q_{1}>\frac{\left(r e^{-d_{1} \tau}-d_{2}\right)\left(e^{d_{3} T_{k}}-1\right)}{\beta} \doteq \overline{q_{1 *}}
$$

which means that if only infective pests are released impulsively, then the release amount must be larger than $\overline{q_{1 *}}$ to ensure the eradication of the susceptible pest. 
Through Theorem 11 and Corollary 12, we can get the sufficient conditions for the susceptible pests-eradication periodic solution; that is, the susceptible pest population is eradicated totally. But in practice, from the view point of keeping ecosystem balance and preserving biological resources, it is not necessary to eradicate the susceptible pest population. In fact we hope the susceptible pests and enemies can coexist when the susceptible pests do not cause immense economic loss. Thus, it is necessary to discuss the permanence of system (6).

Theorem 13. System (6) is permanent provided that

$$
r e^{-d_{1} \tau}>d_{2}+\frac{\beta q_{1}}{1-e^{d_{3} T_{k}}}+\frac{a q_{2} \sum_{j=1}^{j=p} e^{-d_{4}\left(\lambda_{p}-\lambda_{j}\right)}}{1-e^{-d_{4} T_{k}}} .
$$

Proof. Since (65) holds, we can choose sufficiently small positive number $\epsilon, h_{4}\left(h_{4}<\min \left(d_{3} / \beta, d_{4} /\left(\delta a-b d_{4}\right)\right)\right.$ such that

$$
\begin{aligned}
\eta= & r e^{-d_{1} \tau}-d_{2}-\alpha h_{4}-\beta\left(\frac{q_{1}}{1-e^{\left(\beta h_{4}-d_{3}\right) T_{k}}}+\epsilon\right) \\
& -a\left(\frac{q_{2} \sum_{j=1}^{j=p} e^{\left(\left(\delta a h_{4} /\left(1+b h_{4}\right)\right)-d_{4}\right)\left(\lambda_{p}-\lambda_{j}\right)}}{1-e^{\left(\left(\delta a h_{4} /\left(1+b h_{4}\right)\right)-d_{4}\right) T_{k}}}+\epsilon\right)>0 .
\end{aligned}
$$

Suppose $(S(t), I(t), y(t))$ is any solution of system (6) with initial values (7). By Lemma 4, there exists a positive constant $M$ such that $S(t)<M, I(t)<M, y(t)<M$ for $t$ large enough. From (56), we know that $I(t), y(t)$ are positively lower bounded.
Thus we only need to find $h_{3}>0$ such that $S(t)>h_{3}$ for $t$ large enough. We claim that for any $t_{0}>0$, it is impossible that $S(t)<h_{4}$ for all $t \geq t_{0}$. Suppose that the claim is not true. Then there is a $t_{0}>0$ such that $S(t)<h_{4}$ for all $t \geq t_{0}$. Then, from system (6) for all $t \geq t_{0}$ we have

$$
\begin{aligned}
& \dot{I}(t)=\beta S(t) I(t)-d_{3} I(t) \\
& \leq\left(\beta h_{4}-d_{3}\right) I(t), \\
& \dot{y}(t)= \frac{\delta a S(t)}{1+b S(t)} y(t)-d_{4} y(t) \\
& \leq\left(\frac{\delta a h_{4}}{1+b h_{4}}-d_{4}\right) y(t), \\
& \Delta y(t)=q_{2}, \quad t=\tau_{n}+\lambda_{i}, i \in P \\
& t \neq \tau_{n}, \\
& t \neq \tau_{n}+\lambda_{i}, \\
& \Delta I(t)=q_{1}, \quad t=\tau_{n}, n \in z_{+} .
\end{aligned}
$$

From Lemmas 3 and 4, for the above selected $\epsilon>0$, there exists a time $T_{1}>t_{0}+\tau$ such that

$$
\begin{gathered}
I(t) \leq \varphi_{1}^{*}(t)+\epsilon<\frac{q_{1}}{1-e^{\left(\beta h_{4}-d_{3}\right) T_{k}}}+\epsilon=\eta_{1}, \\
y(t) \leq \varphi_{2}^{*}(t)+\epsilon<\frac{q_{2} \sum_{j=1}^{j=p} e^{\left(\left(\delta a h_{4} /\left(1+b h_{4}\right)\right)-d_{4}\right)\left(\lambda_{p}-\lambda_{j}\right)}}{1-e^{\left(\left(\delta a h_{4} /\left(1+b h_{4}\right)\right)-d_{4}\right) T_{k}}}+\epsilon=\eta_{2}
\end{gathered}
$$

hold for all $t \geq T_{1}$, where $\varphi_{1}^{*}(t), \varphi_{2}^{*}(t)$ are the solution of

$$
\begin{aligned}
& \left.\begin{array}{c}
\dot{\varphi}_{1}(t)=\left(\beta h_{4}-d_{3}\right) \varphi_{1}(t), \\
\dot{\varphi}_{2}(t)=\left(\frac{\delta a h_{4}}{1+b h_{4}}-d_{4}\right) \varphi_{2}(t),
\end{array}\right\} t \neq \tau_{n}, t \neq \tau_{n}+\lambda_{i}, \\
& \Delta \varphi_{2}(t)=q_{2}, \quad t=\tau_{n}+\lambda_{i}, i \in P, \\
& \Delta \varphi_{1}(t)=\mathrm{q}_{1}, \quad t=\tau_{n}, n \in z_{+}, \\
& \varphi_{1}\left(T_{1}^{+}\right)=I\left(T_{1}^{+}\right)>0, \quad \varphi_{2}\left(T_{1}^{+}\right)=y\left(T_{1}^{+}\right)>0, \\
& \varphi_{1}^{*}(t)=\varphi_{1}^{*} e^{\left(\beta h_{4}-d_{3}\right)\left(t-\tau_{n}\right)}, \quad t \in\left(\tau_{n}, \tau_{n+1}\right], \quad \varphi_{1}^{*}=\frac{q_{1}}{1-e^{\left(\beta h_{4}-d_{3}\right) T_{k}}}, \\
& \varphi_{2}^{*}(t)= \begin{cases}\varphi_{2}^{*} e^{\left(\left(\delta a h_{4} /\left(1+b h_{4}\right)\right)-d_{4}\right)\left(t-\tau_{n}\right)}, \quad t \in\left(\tau_{n}, \tau_{n}+\lambda_{1}\right], & \\
\varphi_{2}^{*} e^{\left(\left(\delta a h_{4} /\left(1+b h_{4}\right)\right)-d_{4}\right)\left(t-\tau_{n}\right)}+q_{2} \sum_{j=1}^{j=i} e^{\left(\left(\delta a h_{4} /\left(1+b h_{4}\right)\right)-d_{4}\right)\left(t-\tau_{n}-\lambda_{j}\right)}, & t \in\left(\tau_{n}+\lambda_{i}, \tau_{n}+\lambda_{i+1}\right], \\
\vdots & \\
\varphi_{2}^{*} e^{\left(\left(\delta a h_{4} /\left(1+b h_{4}\right)\right)-d_{4}\right)\left(t-\tau_{n}\right)}+q_{2} \sum_{j=1}^{j=p} e^{\left(\left(\delta a h_{4} /\left(1+b h_{4}\right)\right)-d_{4}\right)\left(t-\tau_{n}-\lambda_{j}\right)}, & t \in\left(\tau_{n}+\lambda_{p}, \tau_{n+1}\right],\end{cases} \\
& \varphi_{2}^{*}=\frac{q_{2} \sum_{j=1}^{j=p} e^{\left(\left(\delta a h_{4} /\left(1+b h_{4}\right)\right)-d_{4}\right)\left(T_{k}-\lambda_{j}\right)}}{1-e^{\left(\left(\delta a h_{4} /\left(1+b h_{4}\right)\right)-d_{4}\right) T_{k}}} .
\end{aligned}
$$


Note that the first equation of system (6) can be rewritten as

$$
\begin{aligned}
\dot{S}(t)= & r e^{-d_{1} \tau} S(t-\tau)-d_{2} S(t)-\alpha S^{2}(t) \\
& -\beta S(t) I(t)-\frac{a S(t)}{1+b S(t)} y(t) \\
= & {\left[r e^{-d_{1} \tau}-d_{2}-\alpha S(t)-\beta I(t)-\frac{a y(t)}{1+b S(t)}\right] S(t) } \\
& -r e^{-d_{1} \tau} \frac{d}{d t}\left(\int_{t-\tau}^{t} S(u) d u\right) .
\end{aligned}
$$

In the following we define a Liapunov functional as

$$
V(t)=S(t)+r e^{-d_{1} \tau} \int_{t-\tau}^{t} S(u) d u .
$$

Then the derivative of $V(t)$ with respect to the solution of system (6) is

$$
\begin{aligned}
\left.\dot{V}(t)\right|_{(6)}= & r e^{-d_{1} \tau} S(t)-d_{2} S(t)-\alpha S^{2}(t) \\
& -\beta S(t) I(t)-\frac{a S(t)}{1+b S(t)} y(t) \\
= & {\left[r e^{-d_{1} \tau}-d_{2}-\alpha S(t)-\beta I(t)-\frac{a y(t)}{1+b S(t)}\right] S(t) . }
\end{aligned}
$$

From (68) and (72) we have

$$
\begin{aligned}
\left.\dot{V}(t)\right|_{(6)}= & {\left[r e^{-d_{1} \tau}-d_{2}-\alpha S(t)-\beta I(t)-\frac{a y(t)}{1+b S(t)}\right] S(t) } \\
\geq & {\left[r e^{-d_{1} \tau}-d_{2}-\alpha S(t)-\beta\left(\varphi_{1}^{*}(t)+\epsilon\right)\right.} \\
& \left.-a\left(\varphi_{2}^{*}(t)+\epsilon\right)\right] S(t) .
\end{aligned}
$$

Let $S_{0 *}=\min _{t \in\left[T_{1}, T_{1}+\tau\right]} S(t)>0$.

We will show that $S(t) \geq S_{0 *}$ for all $t>T_{1}$. Otherwise there exists a nonnegative constant $T_{2}$ such that $S(t) \geq S_{0 *}$ for $t \in\left[T_{1}, T_{1}+\tau+T_{2}\right], S\left(T_{1}+\tau+T_{2}\right)=S_{0 *}$ and $\dot{S}\left(T_{1}+\tau+T_{2}\right) \leq 0$. Thus from the first equation of system (6) and (66) we easily see that

$$
\begin{aligned}
\dot{S}\left(T_{1}+\tau+T_{2}\right)= & r e^{-d_{1} \tau} S\left(T_{1}+T_{2}\right) \\
& -d_{2} S\left(T_{1}+\tau+T_{2}\right)-\alpha S^{2}\left(T_{1}+\tau+T_{2}\right) \\
& -\beta S\left(T_{1}+\tau+T_{2}\right) I\left(T_{1}+\tau+T_{2}\right) \\
& -\frac{a S\left(T_{1}+\tau+T_{2}\right)}{1+b S\left(T_{1}+\tau+T_{2}\right)} y\left(T_{1}+\tau+T_{2}\right) \\
> & S_{0 *}\left[r e^{-d_{1} \tau}-d_{2}-\alpha h_{4}\right. \\
& \left.-\beta\left(\varphi_{1}^{*}(t)+\epsilon\right)-a\left(\varphi_{2}^{*}(t)+\epsilon\right)\right] \\
> & S_{0 *} \eta>0,
\end{aligned}
$$

which is a contradiction. Hence we get that $S(t) \geq S_{0 *}>0$ for all $t \geq T_{1}$. From (68), for all $t>T_{1}$ we have

$$
\begin{aligned}
\dot{V}(t)= & r e^{-d_{1} \tau} S(t)-d_{2} S(t)-\alpha S^{2}(t) \\
& -\beta S(t) I(t)-\frac{a S(t)}{1+b S(t)} y(t), \\
= & {\left[r e^{-d_{1} \tau}-d_{2}-\alpha S(t)-\beta I(t)-\frac{a y(t)}{1+b S(t)}\right] S(t) } \\
> & S_{0 *}\left[r e^{-d_{1} \tau}-d_{2}-\alpha h_{4}-\beta\left(\varphi_{1}^{*}(t)+\epsilon\right)\right. \\
& \left.\quad-a\left(\varphi_{2}^{*}(t)+\epsilon\right)\right]
\end{aligned}
$$$$
>\mathrm{S}_{0 *} \eta>0
$$

which implies $V(t) \rightarrow \infty$ as $t \rightarrow \infty$. This is a contradiction to $V(t) \leq M+r \tau M e^{-d_{1} \tau}$. Therefore for any positive constant $t_{0}$ the inequality $S(t)<h_{4}$ cannot hold for all $t \geq t_{0}$.

By the claim, we need to consider two cases.

Case 1. $S(t)>h_{4}$ for all large $t$, then our aim is obtained.

Case 2. $S(t)$ oscillates about $h_{4}$ for all large $t$. Denote

$$
h_{3}=\min \left(\frac{h_{4}}{2}, h_{4} e^{-\left(d_{2}+\alpha M+\beta \eta_{2}+a \eta_{2}\right) \tau}\right) .
$$

In the following we will show that $S(t)>h_{3}$. Since $S(t)$ oscillates about $h_{4}$, there exist positive constants $\bar{t}$ and $\theta$, such that

$$
\begin{gathered}
S(\bar{t})=S(\bar{t}+\theta)=h_{4}, \\
S(t)<h_{4}, \quad \bar{t}<t<\bar{t}+\theta,
\end{gathered}
$$

where $\bar{t}$ is sufficiently large, and the inequalities (68) hold for $\bar{t} \leq t \leq \bar{t}+\theta$. Since $S(t)$ is continuous and bounded, and it is not effected by impulse, there exists a constant $T_{3}$ (where $0<T_{3}<\tau$ and $T_{3}$ is independent of the choice of $\bar{t}$ ) such that $S(t)>h_{4} / 2$ for all $\bar{t}<t<\bar{t}+T_{3}$.

If $\theta<T_{3}$, then our aim is obtained. have

If $T_{3}<\theta<\tau$, from the first equation of system (6) we

$$
\begin{aligned}
\dot{S}(t) & \geq\left(-d_{2}-\alpha S(t)-\beta I(t)-a y(t)\right) S(t), \\
& \geq-\left(d_{2}+\alpha M+\beta \eta_{1}+a \eta_{2}\right) S(t) \quad \text { for } \bar{t} \leq t \leq \bar{t}+\theta,
\end{aligned}
$$

and then we have

$$
S(t) \geq h_{4} e^{-\left(d_{2}+\alpha M+\beta \eta_{1}+a \eta_{2}\right) \tau}, \quad \text { for } \bar{t} \leq t \leq \bar{t}+\theta<\bar{t}+\tau,
$$

and it is clear that $S(t) \geq h_{3}$ for $\bar{t} \leq t \leq \bar{t}+\theta$.

If $\theta>\tau$, then we have that $S(t) \geq h_{3}$ for $\bar{t} \leq t \leq \bar{t}+\tau$. Then, proceeding exactly as the proof for above claim, we see that $S(t) \geq h_{3}$ for $\bar{t}+\tau \leq t \leq \bar{t}+\theta$. Since this kind of interval $[\bar{t}, \bar{t}+\theta]$ 
is arbitrarily chosen (we only need $\bar{t}$ to be large), we can get that $S(t) \geq h_{3}$ for all $t$ large enough. In view of our arguments above, the choice of $h_{3}$ is independent of the positive solution of system (6) which satisfies that $S(t) \geq h_{3}$ for sufficiently large $t$. This completes the proof of the theorem.

Corollary 14. (1) If $q_{1}=0$, then condition (65) of Theorem 13 becomes

$$
q_{2}<\frac{\left(r e^{-d_{1} \tau}-d_{2}\right)\left(1-e^{-d_{4} T_{k}}\right)}{a \sum_{j=1}^{j=p} e^{-d_{4}\left(\lambda_{p}-\lambda_{j}\right)}}=\overline{q_{2 *}},
$$

which means that if only natural enemies are released impulsively, and the release amount is less than $\overline{q_{2 *}}$, then system (6) is permanent. That is, the pest and natural enemy will coexist.

(2) If $q_{2}=0$, then the condition (65) of Theorem 13 becomes

$$
q_{1}<\frac{\left(r e^{-d_{1} \tau}-d_{2}\right)\left(1-e^{d_{3} T_{k}}\right)}{\beta}=\overline{q_{1 *}},
$$

which means that if only infective pests are released impulsively, and the release amount is less than $\overline{q_{1 *}}$, then system (6) is permanent. That is, the pest and natural enemy will coexist.

\section{Discussion}

In this paper, a stage-structured predator-prey model with disease in the prey is considered. The prey stands for the pest population, and the predator stands for the natural enemy population. For the purpose of integrated pest management, two types of impulsive control strategies are used. In Case 1 , infective prey applications are more frequent than releases of natural enemies. In Case 2, natural enemies releases are more frequent than releases of infected prey. In both cases, we analyzed the global attractivity of the susceptible pesteradication periodic solution of the systems, and we also obtained the condition for the permanence of the systems.

In Section 4, the first case is analyzed. By Theorem 7, the sufficient condition for the global attraction of the susceptible pest-eradication periodic solution is obtained, which means that if the release amount of infective pest and natural enemy satisfy certain conditions, then the susceptible pest will be doomed. By the result of Theorem 9, the sufficient condition for the permanence of system (5) is also obtained, which means that the pest and the natural enemy will coexist for all time, if the release amounts of infective pest and natural enemy meet some critical values. Corollary 8 and 10 show that if only one control measure is taken, that is, either only infective pests are released or only natural enemies are released, then the release amount must satisfy certain conditions.

In Section 5, we use similar method to analyze the second case. In Theorem 11, the sufficient condition for the global attraction of the susceptible pest-eradication periodic solution is obtained, which means that if the release amounts of infective pest and natural enemy meet certain conditions, then the susceptible pest will be doomed. By the result of Theorem 13, the sufficient condition for the permanence of system (6) is also obtained, which means that the pest and the natural enemy will coexist for all time, if the release amounts of infective pest and natural enemy meet some critical values. Corollary 12 and 14 show that if only one measure is taken, that is, either only infective pests are released or only natural enemies are released, then the release amount must satisfy certain conditions.

From the above theoretical results obtained in Sections 4 and 5, we can choose different control strategies to control pest. We can do it by releasing infected pest more frequently than releasing natural enemy, or vice versa. However, in practice, every control measure needs certain cost, and we must think about the cost before adopting a measure. For example, the infective prey (pest) being cultivated in the laboratory and the natural enemy (predator) being migrated from other regions all need some cost. We can choose different pest management methods with different costs. In both models, different parameters mean different cost for control measures. Therefore, we have an interesting problem. In the view point of economy, under the premise of controlling the pest, which kind of control method is more suitable? This will be an optimal control problem, and we leave it as our future work.

\section{Acknowledgments}

The authors would like to thank the referees for their helpful suggestions, which improved the quality of this paper greatly. The first author is supported by Postdoctoral Science Foundation of China (no. 2011M501428). The second author is supported by NNSF of China (nos. 11171199, 81161120403).

\section{References}

[1] R. Shi and L. Chen, "An impulsive predator-prey model with disease in the prey for integrated pest management," Communications in Nonlinear Science and Numerical Simulation, vol. 15, no. 2, pp. 421-429, 2010.

[2] R. Shi, X. Jiang, and L. Chen, "A predator-prey model with disease in the prey and two impulses for integrated pest management," Applied Mathematical Modelling, vol. 33, no. 5, pp. 2248-2256, 2009.

[3] Y. Pei, X. Ji, and C. Li, "Pest regulation by means of continuous and impulsive nonlinear controls," Mathematical and Computer Modelling, vol. 51, no. 5-6, pp. 810-822, 2010.

[4] J. Yang and S. Tang, "Effects of population dispersal and impulsive control tactics on pest management," Nonlinear Analysis: Hybrid Systems, vol. 3, no. 4, pp. 487-500, 2009.

[5] Y. Shao and B. Dai, "The dynamics of an impulsive delay predator-prey model with stage structure and Beddington-type functional response," Nonlinear Analysis: Real World Applications, vol. 11, no. 5, pp. 3567-3576, 2010.

[6] H. Zhang, P. Georgescu, and L. Chen, "An impulsive predatorprey system with Beddington-DeAngelis functional response and time delay," International Journal of Biomathematics, vol. 1, no. 1, pp. 1-17, 2008.

[7] C. Wei and L. Chen, "Eco-epidemiology model with age structure and prey-dependent consumption for pest management," Applied Mathematical Modelling, vol. 33, no. 12, pp. 4354-4363, 2009.

[8] S. Tang, G. Tang, and R. A. Cheke, "Optimum timing for integrated pest management: modelling rates of pesticide 
application and natural enemy releases," Journal of Theoretical Biology, vol. 264, no. 2, pp. 623-638, 2010.

[9] B. Liu, Q. Zhang, and Y. Gao, "The dynamics of pest control pollution model with age structure and time delay," Applied Mathematics and Computation, vol. 216, no. 10, pp. 2814-2823, 2010.

[10] J. Liang and S. Tang, "Optimal dosage and economic threshold of multiple pesticide applications for pest control," Mathematical and Computer Modelling, vol. 51, no. 5-6, pp. 487-503, 2010.

[11] F. L. McEwen and R. G. Stephenson, The Use and Significance of Pesticides in the Environment, John Wiley \& Sons, New York, NY, USA, 1979.

[12] W. Phelps, Pesticide Environmental Fate: Bridging the Gap Between Laboratory and Field Studies, American Chemical Society, Washington, DC, USA, 2002.

[13] P. Georgescu and G. Morosanu, "Pest regulation by means of impulsive controls," Applied Mathematics and Computation, vol. 190, no. 1, pp. 790-803, 2007.

[14] G. Pang and L. Chen, "Dynamic analysis of a pest-epidemic model with impulsive control," Mathematics and Computers in Simulation, vol. 79, no. 1, pp. 72-84, 2008.

[15] Z. Xiang, Y. Li, and X. Song, "Dynamic analysis of a pest management SEI model with saturation incidence concerning impulsive control strategy," Nonlinear Analysis: Real World Applications, vol. 10, no. 4, pp. 2335-2345, 2009.

[16] S. Sun and L. Chen, "Mathematical modelling to control a pest population by infected pests," Applied Mathematical Modelling, vol. 33, no. 6, pp. 2864-2873, 2009.

[17] L. Wang, L. Chen, and J. J. Nieto, “The dynamics of an epidemic model for pest control with impulsive effect," Nonlinear Analysis: Real World Applications, vol. 11, no. 3, pp. 1374-1386, 2010.

[18] H. Zhang, L. Chen, and J. J. Nieto, "A delayed epidemic model with stage-structure and pulses for pest management strategy," Nonlinear Analysis: Real World Applications, vol. 9, no. 4, pp. 1714-1726, 2008.

[19] W. Gao and S. Tang, "The effects of impulsive releasing methods of natural enemies on pest control and dynamical complexity," Nonlinear Analysis: Hybrid Systems, vol. 5, no. 3, pp. 540-553, 2011.

[20] H. I. Freedman, M. Agarwal, and S. Devi, "Analysis of stability and persistence in a ratio-dependent predator-prey resource model," International Journal of Biomathematics, vol. 2, no. 1, pp. 107-118, 2009.

[21] J. Jiao, L. Chen, and S. Cai, "A delayed stage-structured Holling II predator-prey model with mutual interference and impulsive perturbations on predator," Chaos, Solitons and Fractals, vol. 40, no. 4, pp. 1946-1955, 2009.

[22] J. Jiao, X. Meng, and L. Chen, "A stage-structured Holling mass defence predator-prey model with impulsive perturbations on predators," Applied Mathematics and Computation, vol. 189, no. 2, pp. 1448-1458, 2007.

[23] J. Hui and D. Zhu, "Dynamic complexities for prey-dependent consumption integrated pest management models with impulsive effects," Chaos, Solitons and Fractals, vol. 29, no. 1, pp. 233251, 2006

[24] S. Tang and R. A. Cheke, "Models for integrated pest control and their biological implications," Mathematical Biosciences, vol. 215, no. 1, pp. 115-125, 2008.

[25] P. Georgescu and H. Zhang, "An impulsively controlled predator-pest model with disease in the pest," Nonlinear Analysis: Real World Applications, vol. 11, no. 1, pp. 270-287, 2010.
[26] J. Jiao, S. Cai, and L. Chen, "Analysis of a stage-structured predatory-prey system with birth pulse and impulsive harvesting at different moments," Nonlinear Analysis: Real World Applications, vol. 12, no. 4, pp. 2232-2244, 2011.

[27] F. Wang and G. Pang, "The global stability of a delayed predatorprey system with two-stage structure," Chaos, Solitons and Fractals, vol. 40, no. 2, pp. 778-785, 2009.

[28] W. Fengying and W. Ke, "Permanence of variable coefficients predator-prey system with stage structure," Applied Mathematics and Computation, vol. 180, no. 2, pp. 594-598, 2006.

[29] Z. Ma, J. Yang, and G. Jiang, "Impulsive control in a stage structure population model with birth pulses," Applied Mathematics and Computation, vol. 217, no. 7, pp. 3453-3460, 2010.

[30] H. Guo and L. Chen, "A study on time-limited control of singlepest with stage-structure," Applied Mathematics and Computation, vol. 217, no. 2, pp. 677-684, 2010.

[31] R. Xu, M. A. J. Chaplain, and F. A. Davidson, "A Lotka-Volterra type food chain model with stage structure and time delays," Journal of Mathematical Analysis and Applications, vol. 315, no. 1, pp. 90-105, 2006.

[32] J. Jiao, X. Meng, and L. Chen, "Harvesting policy for a delayed stage-structured Holling II predator-prey model with impulsive stocking prey," Chaos, Solitons and Fractals, vol. 41, no. 1, pp. 103-112, 2009.

[33] X. Meng, J. Jiao, and L. Chen, "The dynamics of an age structured predator-prey model with disturbing pulse and time delays," Nonlinear Analysis: Real World Applications, vol. 9, no. 2, pp. 547-561, 2008.

[34] P. Wang, J. Lu, and M. J. Ogorzalek, "Global relative parameter sensitivities of the feed-forward loops in genetic networks," Neurocomputing, vol. 78, no. 1, pp. 155-165, 2012.

[35] J. Lu and G. Chen, "A time-varying complex dynamical network model and its controlled synchronization criteria," IEEE Transactions on Automatic Control, vol. 50, no. 6, pp. 841-846, 2005.

[36] J. Zhou, J. Lu, and J. Lu, "Adaptive synchronization of an uncertain complex dynamical network," IEEE Transactions on Automatic Control, vol. 51, no. 4, pp. 652-656, 2006.

[37] W. Yu, G. Chen, and J. Lu, "On pinning synchronization of complex dynamical networks," Automatica, vol. 45, no. 2, pp. 429-435, 2009.

[38] J. Lu, X. Yu, G. Chen, and D. Cheng, "Characterizing the synchronizability of small-world dynamical networks," IEEE Transactions on Circuits and Systems I, vol. 51, no. 4, pp. 787796, 2004.

[39] X. Song and L. Chen, "Optimal harvesting and stability for a two-species competitive system with stage structure," Mathematical Biosciences, vol. 170, no. 2, pp. 173-186, 2001.

[40] V. Lakshmikantham, D. D. Bainov, and P. S. Simeonov, Theory of Impulsive Differential Equations, vol. 6 of Series in Modern Applied Mathematics, World Scientific, Singapore, 1989. 


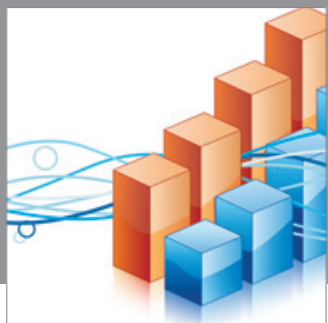

Advances in

Operations Research

mansans

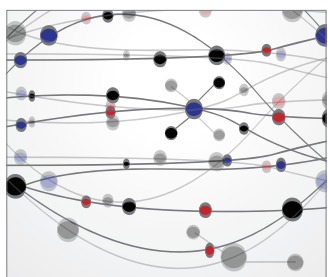

The Scientific World Journal
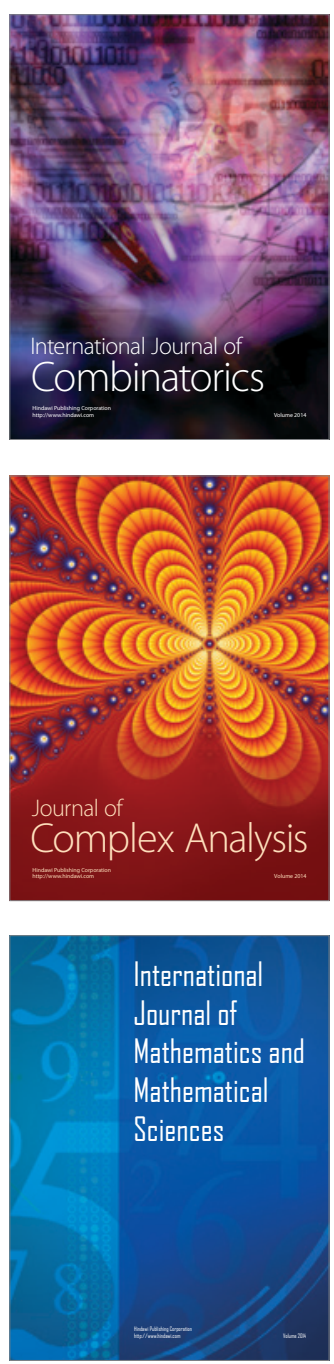
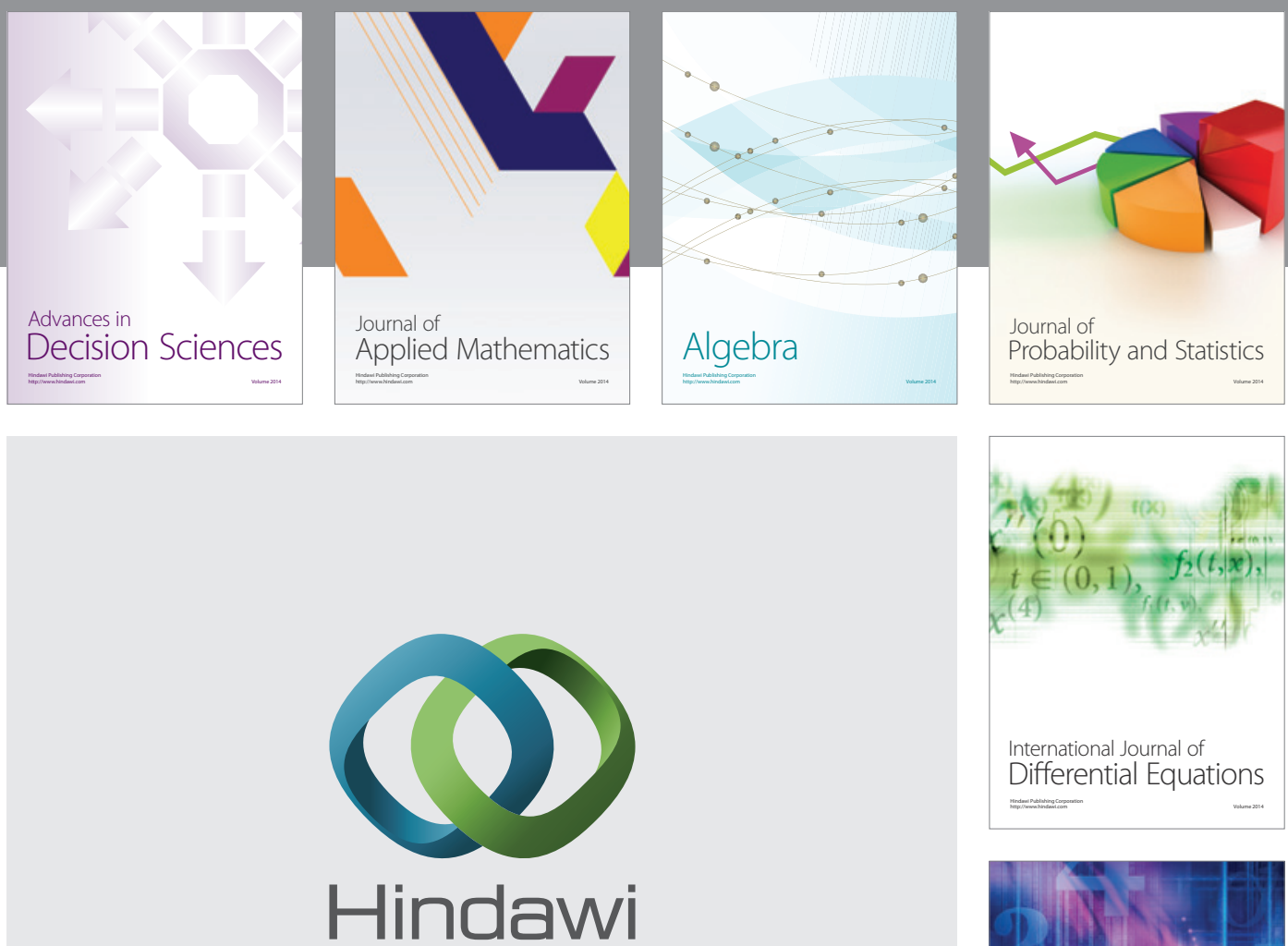

Submit your manuscripts at http://www.hindawi.com
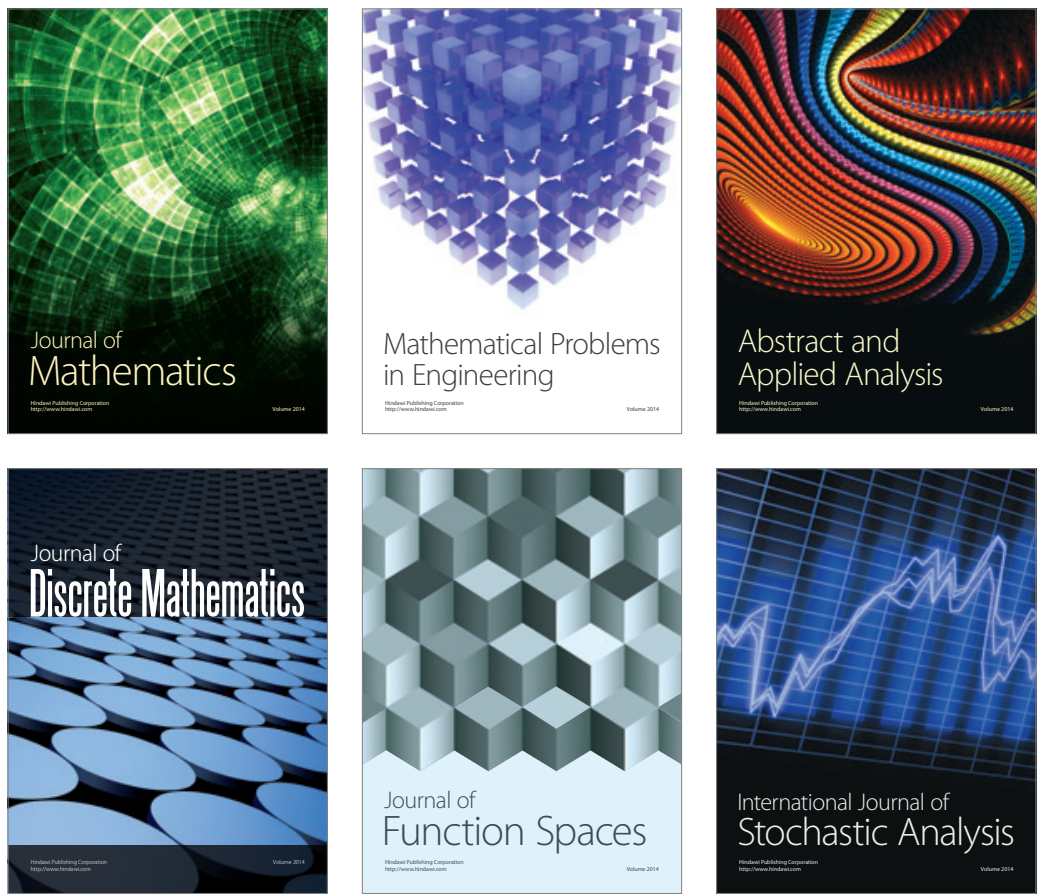

Journal of

Function Spaces

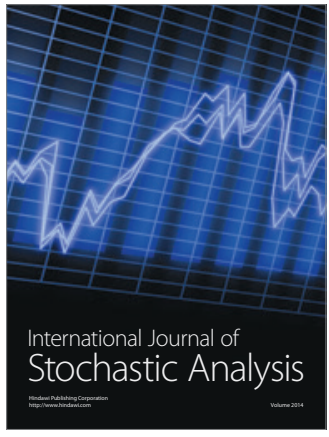

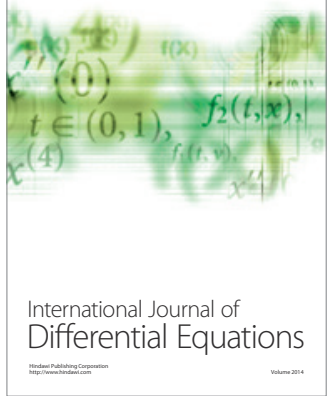
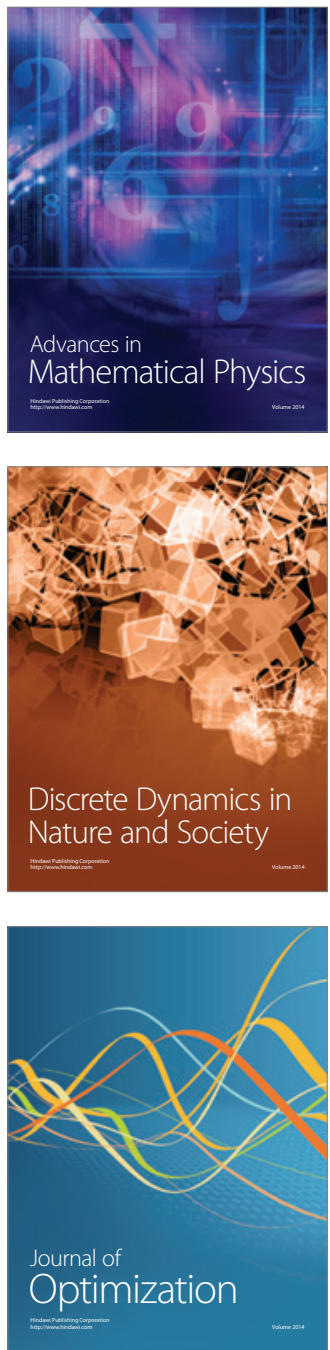